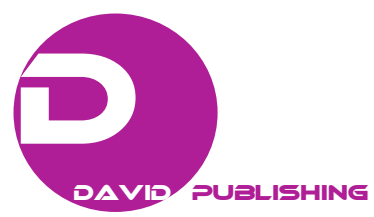

\title{
Case-Report: More than Meets the Leg
}

\author{
Karin Marieke Eggink \\ Radboud University Medical Centre, Nijmegen 6500, Netherlands
}

Received: February 25, 2015 / Accepted: March 1, 2015 / Published: March 31, 2015.

\section{Case}

The authors describe the presentation of a 61-year old woman, with the acute development of a swollen, red-blue and painful leg. When the patient got up in the morning she noticed her right leg to be painful, swollen and with a red-blue discoloration. Walking was difficult, but manageable. When she got up $4 \mathrm{~h}$ previous to go to the bathroom she hadn't noticed any problems with her leg.

In the course of two hours the pain and swelling progressed, after which the patient contacted her general practitioner who referred her to the ED (emergency department).

On arrival in the ED the pain had subsided after the use of 1 gram of paracetamol. The patient reported no fever, no wounds or leg trauma of any kind and no signs of respiratory problems, painful breathing, coughing or hemoptysis. Twelve days previous she was admitted for a day in the hospital to undergo minor surgery on her hand. Afterwards she had been in good health until the present day.

Physical examination showed normal vital signs with a pulse of $95 / \mathrm{min}$, tension $126 / 60$, oxygen saturation $96 \%$ and a temperature of $37.0{ }^{\circ} \mathrm{C}$. Examination of the heart, lungs and abdomen showed no abnormal findings.

The right leg was significantly swollen from the toes to the groin area, with $5 \mathrm{~cm}$ in circumferential difference in respect to the left leg. The right leg showed a red discoloration with a cyanotic aspect of

\footnotetext{
Corresponding author: Karin Marieke Eggink, M.D., research field: emergency medicine. E-mail: k.m.eggink@hotmail.com.
}

the foot, the right foot was slightly cooler than left. The arteria dorsalis pedis was adequately palpable on both feet and pitting edema was present. No wounds or other signs of trauma were visible. The whole leg had a tight aspect on palpation and was mildly painful.

An ultrasound of the deep veins was performed, which showed complete obstruction of the deep venous system in all the deep veins, from the vena saphena magna to the vena iliaca externa.

After the ultrasound on re-evaluation the arteria dorsalis pedis was no longer detectable by palpation or doppler.

The differential diagnosis is phlegmasia cerulea dolens. A massive form of deep venous thrombosis of

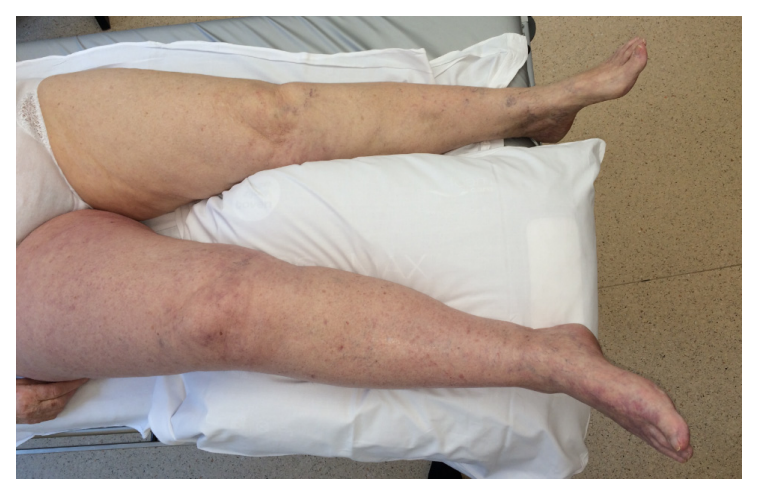

Fig 1 Swelling and discoloration of the whole leg in phlegmasia cerulea dolens.

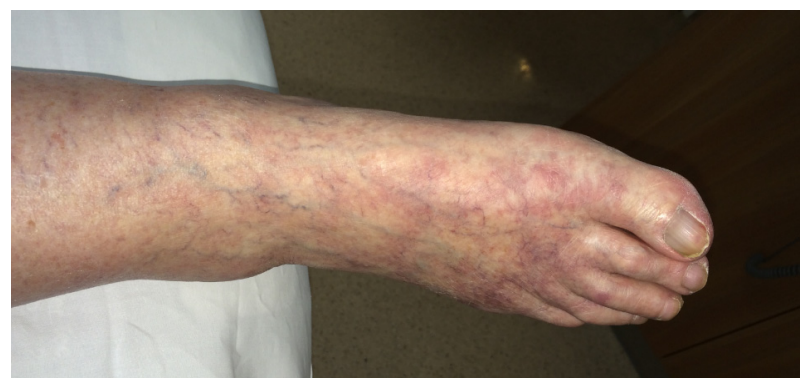

Fig 2 Swelling and cyanosis of the foot in phlegmasia cerulea dolens. 
the lower extremity, which can result in edema, cyanosis, venous gangrene, arterial compromise and compartment syndrome. Phlegmasia cerulea dolens may result in loss of the extremity and or death by circulatory collapse and shock.

The patient was initially treated with 5,000 units of iv heparin to prevent further cloth formation. Following she underwent catheter-directed thrombolysis with Urokinase.

In this case the development of the deep venous thrombosis seems to be idiopathic. The minor hand surgery 12 days previous cannot be considered a risk factor for development of deep venous thrombosis of the leg. A CT-scan of the abdomen showed no signs of a tumor but an anatomical variant of vena cava inferior with drainage of the iliacal system through the lumbal veins and vena ovaricae.

\section{Conclusion}

Phlegmasia cerulea dolens is a limb and life threatening emergency, which must be recognized as soon as possible. Immediate treatment with heparin iv should be started to prevent further cloth formation. Consecutively catheter-directed thrombolysis or surgical trombectomy should be considered, too safe the leg and possibly the life of the patient. 